Artículo de investigación E08A12 - Proyecto "Indicador de desempeño fiscal como herramienta de desarrollo económico local”. Universidad Católica de Oriente * Recibido: 29-01-2016. * Aprobado versión final: 04-11-2016 JEL: E62, H72, H73, H83. * Pp. 61-79

\title{
Indicador de desempeño fiscal: \\ Análisis de los resultados de su aplicación en los municipios del altiplano del oriente antioqueño.
}

\author{
Fiscal performance indicator: \\ Analysis of results of its measures in the East Antioquia \\ Highland municipalities.
}

Carolina Rincón Zapata - Alba Lucía Restrepo

\section{COLOMBIA}

Resumen: Se busca enunciar los factores que marcan la diferencia en la viabilidad financiera en los nueve municipios del Altiplano del Oriente Antioqueño (AOA). En Colombia, la viabilidad financiera de las entidades territoriales se mide mediante el indicador de desempeño fiscal determinado por el Departamento Nacional de Planeación, a partir del cual se otorgan unas tipologías que denotan el estado de la gestión de las finanzas públicas. El método aplicado fue inductivodeductivo, porque se partió de la gestión fiscal del municipio de Rionegro para elaborar recomendaciones aplicables a los demás municipios. Se encontró en general un buen desempeño fiscal, porque ocho de los nueve municipios están clasificados entre las tipologías de solvencia y sostenibilidad. Al final, se ofrecen recomendaciones para el mejoramiento de las condiciones de viabilidad financiera de los municipios.

Palabras claves: finanzas públicas; gestión financiera; indicador de desempeño fiscal; viabilidad financiera.

Abstract: It seeks to state the factors that make a difference in the financial viability in the nine municipalities in eastern Antioquia Altiplano (AOA) are. In Colombia, the financial viability of Local Authorities is measured by the indicator of fiscal performance determined by the National Planning Department, from which typologies that show the status of the management of public finances are granted. The method used was inductive-deductive because we started from the fiscal management of the municipality of Rionegro, to develop recommendations to the other towns of the AOA. It was found that in the territorial entities AOA fiscal performance is evident, because eight of the nine municipalities rank among the types of solvency and sustainability. Then the papers ends up with recommendations for improving the conditions of financial viability.

Keywords: Public Finances; Financial Management; Fiscal Performance Indicator; Financial Viability.

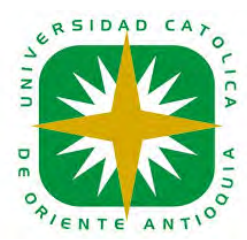

Carolina Rincón Zapata es Economista, especialista en Finanzas con énfasis en Banca de Inversión, Magíster en Economía, docente de tiempo completo en la Facultad de Ciencias Económicas y Administrativas, Universidad Católica de Oriente (Rionegro, Antioquia). Contacto: crincon@uco.edu.co.

Alba Lucía Restrepo es Economista, Magíster en Gobierno, docente de tiempo completo en la Facultad de Ciencias Económicas y Administrativas, Universidad Católica de Oriente (Rionegro, Antioquia). Contacto: arestrepo@uco.edu.co. 


\title{
Indicador de desempenho fiscal:
}

Análise dos resultados da sua aplicação nos municipios do planalto do leste da Antioquia.

\begin{abstract}
Resumo: Procuramos enunciar quais são os fatores que marcam a diferença na viabilidade financeira nos nove municípios do Planalto do Oriente Antioqueño (AOA). Na Colômbia, a viabilidade financeira das Entidades Territoriais mede-se mediante o indicador de desempenho fiscal, determinado pelo Departamento Nacional de Planejamento, a partir do qual se outorgam umas tipologias que denotam o estado da gestão das finanças públicas. O método aplicado foi indutivo-dedutivo porque se partiu de observar a gestão fiscal do Município de Rionegro, para desenvolver recomendações que possam ser aplicadas a outros municípios do AOA. Encontrou-se que nas entidades territoriais do AOA se evidencia um bom desempenho fiscal, porque oito dos nove municípios estão classificados entre as tipologias de solvência e sustentabilidade. Finalmente são oferecidas recomendações para melhorar as condições de viabilidade financeira dos municípios analisados.
\end{abstract}

Palavras-chave: finanças públicas; gestão financeiro; indicadores de desempenho fiscal; viabilidade financeira.

\section{Introducción}

a obtención y gestión de recursos hacen parte fundamental de las actividades económicas que mundialmente se presentan a través de la interacción de los componentes de la demanda, es decir, las familias, las empresas, el gobierno y el resto del mundo. Las finanzas se enfocan precisamente en el estudio de la mejor forma de obtención y manejo de los fondos necesarios para el cumplimiento de los objetivos, ya sea de los individuos, de las organizaciones o del mismo Estado; esta gestión implica la búsqueda de la viabilidad financiera de las operaciones.

En el caso específico del Estado, la viabilidad financiera permite, además de su sostenibilidad en el tiempo, cumplir con los objetivos que por ley se le ha asignado y que son propios de su naturaleza. Esta viabilidad se refleja por medio de unas condiciones fiscales de solvencia, sostenibilidad, vulnerabilidad, riesgo y deterioro, definidas por el Departamento Nacional de Planeación, DNP (2012), y que reflejan para las entidades territoriales la eficiencia en la ejecución del gasto, la generación de ahorro, el mejoramiento de los niveles de inversión, la capacidad para atender el pago de todas sus deudas y que, además, acrediten la gestión responsable de los recursos públicos.

Las anteriores condiciones, además de estar influenciadas por la gestión administrativa que de ellas hagan las entidades territoriales, dependen 
en gran medida de la estructura económica y productiva de estas y no únicamente de los niveles de desarrollo o inversión que posean. En este sentido, los municipios del AOA poseen una estructura productiva y económica similar; estos municipios están localizados en una misma zona geográfica que actualmente presenta destacados niveles de inversión y un desarrollo importante dentro del departamento y del país. No obstante lo anterior, según los datos acerca del desempeño fiscal de los municipios del Oriente Antioqueño, arrojados por el DNP (2013), no todos se encuentran en el mismo nivel de viabilidad financiera: por ejemplo, el municipio de La Unión presenta condiciones de vulnerabilidad, mientras que los municipios de San Vicente Ferrer, El Santuario, Marinilla y El Carmen de Viboral reflejan condiciones de sostenibilidad; a su vez, municipios como La Ceja, Guarne, El Retiro y Rionegro arrojan condiciones de solvencia.

En este sentido, se hace importante analizar a fondo cuáles son las condiciones financieras que llevan a que existan estos diferenciales entre los nueve municipios del AOA. Dicho análisis sirve no solo para determinar los faltantes en la gestión de los entes territoriales, sino para que estos definan estrategias para el mejoramiento o sostenimiento de sus condiciones financieras, posibilitando con ello cumplir sus metas y mejorar la calidad de los bienes públicos y meritorios ofrecidos. Lo anterior trae consigo el beneficio de avanzar gradualmente hacia una mayor autonomía en materia de ingresos para no tener que depender tanto de recursos de transferencias nacionales, condicionadas en su mayor parte a unos fines preestablecidos por la ley.

El presente artículo enuncia cuáles son los factores que marcan la diferencia en la viabilidad financiera, encontrados como resultado de la investigación denominada Indicador de desempeño fiscal como instrumento de gestión del desarrollo económico local' que se desarrolló con el anterior propósito para los municipios del AOA, y de esta forma proponer estrategias para el mejoramiento de las finanzas públicas locales.

\section{Marco teórico}

La Ley 617 de 2000 es una de las normas a las cuales las administraciones locales deben responder en materia de gestión pública. Esta ley establece un seguimiento para los diferentes niveles del Gobierno, lo cual se traduce en mejores resultados de la hacienda y con ello en una

1 Investigación realizada en el marco de la convocatoria de Investigación y Desarrollo de la Universidad Católica de Oriente (Rionegro, Antioquia) para el año 2014. Grupo de Investigación: FACEA. Título: Indicador de desempeño fiscal como herramienta del desarrollo económico local. Número del Proyecto: 151 
mayor gobernabilidad. En lo referente a mejores resultados fiscales, el Departamento Nacional de Planeación (DNP) realiza periódicamente una compilación de información en la que define las condiciones de viabilidad financiera y donde, a su vez, da cuenta de la evolución de los componentes estratégicos de las haciendas locales. Específicamente, el artículo 79 de dicha norma estipula lo siguiente:

Artículo 79. Control social a la gestión pública territorial. EI DNP publicará en medios de amplia circulación nacional con la periodicidad que señale el reglamento y por lo menos una vez al año, los resultados de la evaluación de la gestión de todas las entidades territoriales, incluidos sus organismos de control, según la metodología que se establezca para tal efecto (Ministerio de Hacienda y Crédito Público, 2000, p. 43).

En este sentido, el DNP ha determinado una metodología de evaluación a partir de un indicador sintético, cuyos valores se encuentran en una escala entre 0 y 100 , donde los que son cercanos a cero reflejan un rezago en la administración de los recursos, mientras que valores cercanos a 100 se traducen en logros de la entidad territorial en cuanto a las variables definidas por el indicador de desempeño fiscal, que más adelante serán enunciadas y explicadas.

Por su parte, el DNP ha determinado además unas tipologías para la anterior evaluación, que en última instancia van a ser las que determinan o no la viabilidad financiera de las entidades territoriales, y que a continuación se explican detalladamente según lo establecido por este organismo nacional (DNP, 2012):

- La tipología solvente corresponde a las entidades territoriales cuyo indicador de desempeño fiscal es igual o superior a 80 puntos. Estos municipios, en términos generales, gozan de unas finanzas saludables, indicando una buena gestión de sus ingresos, sus gastos y su endeudamiento. En comparación con el resto, las entidades que se encuentran en esta tipología tienen mejores condiciones de solvencia financiera.

- Las entidades clasificadas como sostenibles son aquellas para las cuales el indicador se sitúa entre 70 y 80 puntos. Su situación es similar a los municipios ubicados en la tipología solvente, pero la magnitud de los indicadores es menor.

- Las vulnerables corresponden a las entidades territoriales cuyo indicador de desempeño fiscal está entre 60 y 70 puntos, lo que significa que -aunque pueden cumplir con los límites de gasto de la 
Ley 617 de 2000 y generar ahorros propios- son dependientes de los recursos de la nación y se les dificulta la generación de excedentes financieros.

- Están en riesgo las entidades con un indicador entre 40 y 60 puntos, las cuáles presentan las mismas características de los municipios vulnerables, pero en un grado superior, por lo que requieren mayor atención para garantizar su sostenibilidad financiera.

- Las entidades que presentan deterioro tienen un indicador menor o igual a 40, lo que refleja baja capacidad de ahorro, dificultades para garantizar el pago de los gastos de funcionamiento, alta dependencia de las transferencias y menores posibilidades de inversión.

- Por último, en la categoría sin información se encuentran las entidades que no reportaron información o que la reportaron incompleta o inconsistente, razón por la cual se hace imposible conocer el desempeño en sus finanzas y por eso ocupan los últimos lugares del escalafón de desempeño fiscal. Estas entidades requieren acción inmediata de los organismos de control.

Como ya se enunció, el DNP define las anteriores condiciones de viabilidad financiera para cada una de las entidades territoriales por medio de un indicador de desempeño fiscal. A continuación, se describen de forma detallada las seis variables que hacen parte de dicho indicador, según información suministrada por José Lenin Galindo Urquijo (comunicación personal, 25 de junio de 2014) y el informe de desempeño fiscal de los departamentos y municipios, elaborado por el DNP (2010):

- Autofinanciamiento de los gastos de funcionamiento: este primer indicador parte de los gastos de funcionamiento que reportan las entidades territoriales, y cuyo porcentaje es establecido a partir del artículo 19 de la Ley 617 de 2000, como marco de referencia en lo relativo al cumplimiento en gestión administrativa por concepto de servicios personales, gastos generales y transferencias corrientes, de acuerdo con la categoría correspondiente. La ley ha establecido lo siguiente:

"Los gastos de funcionamiento de las entidades territoriales deben financiarse con sus ingresos corrientes de libre destinación, de tal manera que estos sean suficientes para atender sus obligaciones corrientes, provisionar el pasivo prestacional y pensional $y$ financiar, al menos parcialmente, la inversión pública autónoma de las mismas" (Ministerio de Hacienda y Crédito Público, 2000, p. 12). 
En este sentido, lo que define la misma ley es que las entidades territoriales, dependiendo de la categoría asignada, no deberán gastar más del porcentaje definido en relación con sus Ingresos Corrientes de Libre Destinación (ICLD), como se muestra en la tabla 1.

Tabla 1. Límite de gastos de funcionamiento con relación a los ICLD para los municipios según su categoría, establecida por el DNP para el año 2013.

\begin{tabular}{|l|c|l|}
\multicolumn{1}{|c|}{ Categoría } & $\begin{array}{c}\text { Porcentaje de Gasto en } \\
\text { relación con los ICLD }\end{array}$ & \multicolumn{1}{|c|}{ Municipios del AOA } \\
\hline $\begin{array}{l}\text { Distrito Capital y Especial } \\
\text { Primera }\end{array}$ & $50 \%$ & \\
Segunda y Tercera & $65 \%$ & $\begin{array}{l}\text { Rionegro } \\
\text { El Carmen de Viboral, } \\
\text { Guarne, La Unión, Marinilla, } \\
\text { El Retiro, San Vicente, El } \\
\text { Santuario, La Ceja }\end{array}$ \\
\hline
\end{tabular}

Fuente: Elaboración propia a partir de la Ley 617 de 2000.

- Magnitud de la deuda: se refiere a los recursos que tienen por objeto el cumplimiento de las obligaciones correspondientes al pago de capital, los intereses y las comisiones originadas en operaciones de crédito público (créditos externos, internos y emisión de bonos). Este indicador se obtiene como la proporción de los ingresos disponibles que están respaldando el servicio de la deuda, que se compone de los intereses, las amortizaciones y los costos financieros de la deuda, con relación a los ingresos disponibles, los cuales están conformados por los ingresos tributarios (impuestos propios), no tributarios (tasas, multas y sanciones, contribuciones, aportes, regalías), al igual que las transferencias para libre destinación y las regalías. Este indicador guarda relación con lo que define la Ley 358 de 1997 y la Ley 819 de 2003 (Congreso de la República), y se espera que la deuda total no supere la capacidad de pago de la entidad ni comprometa su liquidez en el pago de otros gastos. Si las entidades subnacionales no contraen tanta deuda o esta se encuentra a la baja, el dinero que se destinaba para el pago de los intereses y las amortizaciones puede aplicarse al presupuesto de inversión que eleva los indicadores de estabilidad económica, social y política. No obstante lo anterior, considerar crecimientos en niveles de deuda pública cuando este índice es demasiado bajo y las entidades territoriales necesitan más inversión podría conducir a un mayor desarrollo local, siempre y cuando no sobrepase los límites de lo establecido en la ley. 
- Dependencia de transferencias: este indicador financiero se refiere a aquellos recursos que se perciben de otros niveles del Estado, tales como nacionales, departamentales o municipales. Así pues, la dependencia de las transferencias y las regalías mide la importancia que estos recursos tienen en relación con el total de fuentes de recaudo; es decir, indica el peso que tienen estos recursos en el total de ingresos, y su magnitud refleja el grado en el cual las transferencias y regalías se convierten en los recursos fundamentales para financiar el desarrollo territorial. Este indicador está en función del modelo de transferencias intergubernamentales del país, el cual establece mecanismos de compensación de las brechas territoriales y la participación en las rentas nacionales bajo la figura de sistema general de participaciones y de regalías, pero también exige esfuerzo propio por parte de las entidades territoriales, de manera que el desarrollo no se financie exclusivamente a partir de las participaciones en las rentas de la nación, sino también con la contrapartida del esfuerzo fiscal propio.

Según José Lenin Galindo Urquijo (comunicación personal, 25 de junio de 2014), un indicador por encima del 30 \% señala que la entidad territorial posee una alta dependencia de las transferencias, lo que indica que financia sus gastos principalmente con recursos de transferencias de la Nación y regalías. De lo contrario, se identifica el esfuerzo fiscal promovido a través de los recursos propios.

- Generación de recursos propios: este indicador mide el esfuerzo fiscal y de ajuste que realizan los gobiernos territoriales con fuentes propias de ingresos, como lo son los impuestos que generan autónomamente las localidades, y mide la importancia de esta gestión tributaria frente a otras fuentes externas de financiamiento del gasto corriente como las transferencias de libre destinación. Esta es una medida calculada a partir del peso relativo de los ingresos tributarios sobre el total de ingresos corrientes.

- Magnitud de la inversión: este indicador está conformado por el gasto de capital que efectúa la entidad territorial en los diferentes tipos de inversión, como es el gasto social, la infraestructura física, la inversión administrativa, entre otros. El indicador permite cuantificar el grado de inversión que hace la entidad territorial respecto al gasto total. Según José Lenin Galindo Urquijo (comunicación personal, 25 de junio de 2014), se espera que este indicador sea superior al $50 \%$ y tendiente al alza, lo que significaría que más de la mitad del gasto se está destinando con prioridad a la inversión.

- La capacidad de ahorro: este indicador, en cierto sentido, es complementario al de cumplimiento a los límites de gasto y mide 
si las entidades territoriales están realmente generando excedentes para inversión después de cubrir los gastos de funcionamiento de la administración central, atender los compromisos derivados de los acuerdos de reestructuración de pasivos, apropiar los recursos para el pago de los pasivos pensionales y otros gastos corrientes.

La metodología que utiliza el DNP para la determinación del indicador de desempeño fiscal a partir de las anteriores seis variables se basa, entonces, en la determinación del peso que cada una de estas tiene dentro del indicador, por medio de la aplicación de un método estadístico por componentes principales. La contribución de cada variable dentro del indicador varía año tras año dentro de un rango de $16 \%$ a $22 \%$, dependiendo del clima económico nacional y su respectiva estandarización mediante la técnica antes mencionada. José Lenin Galindo Urquijo (comunicación personal, 25 de junio de 2014) señala que la ecuación bajo la cual se estima el indicador es la siguiente:

Desempeño Fiscal ${ }_{i}=\beta_{1} X_{1}+\beta_{2} X_{2}+\beta_{3} X_{3}+\beta_{4} X_{4}+\beta_{5} X_{5}+\beta_{6} X_{6}$

Donde: $i=1,2, \ldots 1101$ municipios; $\beta$ :Ponderaciones estimadas para cada variable; y X:Variables estimadas

Es de anotar que, en algunas ocasiones, el DNP ha determinado la ponderación de las variables antes mencionadas mediante promedios simples para recrear o anticipar el comportamiento de los municipios de acuerdo con sus finanzas.

\section{Metodología}

El método de investigación empleado fue inductivo-deductivo porque se observó específicamente la gestión fiscal del municipio de Rionegro, para elaborar recomendaciones que se pudieran aplicar a los otros municipios del AOA. Es preciso anotar, sin embargo, que se encontró que cada ente territorial tiene sus propias realidades financieras y no es tan fácil para los demás adoptar sus modelos de gestión. El tipo de estudio que se realizó es descriptivo porque se caracterizaron cada una de las variables que componen el indicador de desempeño fiscal, según los resultados arrojados, de los municipios del AOA; y a partir de estos se formularon algunas propuestas de mejoramiento en la gestión fiscal local.

Para dicho análisis se realizó una revisión de la metodología usada por el DNP en su construcción y ponderación del indicador. Posteriormente se consultaron los resultados para cada una de estas variables en los nueve municipios del AOA, con los cuales se construyó una matriz ponderada-comparativa que permitió la identificación de las variables 
que desmejoran el indicador de desempeño en comparación con el municipio de Rionegro, y además condujo a concluir si cada una de estas entidades territoriales para el periodo analizado tuvo la tipología solvente, sostenible, vulnerable, en riesgo o en deterioro. A partir de lo anterior se elaboraron algunas recomendaciones en materia de políticas de recaudo y eficiencia del gasto que conduzcan a la disminución de las brechas diferenciales que contribuyen al detrimento del indicador de desempeño fiscal y el ranking fiscal en cada uno de los municipios.

\section{Resultados}

A partir del Indicador de Desempeño Fiscal, el DNP ordena los puntajes obtenidos para todas las entidades territoriales del país, derivando así un ranking que categoriza de mayor a menor los puntajes obtenidos. Para el año 2014, el municipio de Rionegro -con un indicador de 90.73- ocupa el primer puesto en el orden nacional $y$, como es obvio, frente a los ocho restantes municipios del AOA.

De los municipios analizados, el que ocupa el segundo puesto en la tabla es el municipio de El Retiro, con un indicador de 84.72, seguido de Guarne (82.39), La Ceja (80.40), El Carmen de Viboral (77.65), Marinilla (75.03), El Santuario (74.3), San Vicente Ferrer (70.42) y La Unión $(69,82)$. Del total de los municipios clasificados en el nivel nacional, Bagadó (Chocó) ocupa el lugar 1.101 con un indicador de 31,82; al contrastar este indicador con el más bajo resultado en el AOA, obtenido por el municipio de La Unión, se puede colegir que en realidad este último municipio -que en el orden nacional ocupa la posición 370- no presenta un mal indicador que amenace su solvencia de acuerdo con los criterios establecidos por el DNP.

Según la tipología de viabilidad financiera definida por el DNP, donde se asigna a los máximos niveles de deterioro de las Entidades Territoriales un rango entre 0 a 40 puntos en el indicador de desempeño fiscal, ninguno de los municipios del AOA se encuentra dentro de esta condición. Lo anterior indica que los municipios de esta subregión no tienen comprometidas sus posibilidades de inversión social, no poseen una muy baja capacidad de ahorro, no tienen dificultades para garantizar el pago de los gastos de funcionamiento y no presentan una alta dependencia de las transferencias nacionales.

En este mismo sentido, el DNP asigna un rango entre 40 y 60 puntos en el indicador de desempeño fiscal para representar la tipología de riesgo, en la cual tampoco se ubicó en 2014 ninguno de los municipios analizados. Pero bajo la tipología de vulnerabilidad, donde el rango definido se encuentra entre 60 y 70 puntos, sí se clasifica al municipio de La Unión, porque posee un indicador de 69,82; esto significa que tiene una alta dependencia de las transferencias nacionales. 
Por su parte, los municipios de El Carmen de Viboral, Marinilla, El Santuario y San Vicente Ferrer presentan indicadores que se encuentran en el rango entre 70 y 80 , lo que determina niveles de sostenibilidad. A su vez, los municipios de Rionegro, El Retiro, Guarne y La Ceja reflejan el mejor nivel de viabilidad, que está determinado por un indicador superior a 80 puntos y que declara solvencia para estas entidades territoriales.

Los anteriores valores, entonces, recobran sentido al analizar las variables que los componen. En la tabla 2 se relacionan los resultados para cada uno de los municipios analizados.

Tabla 2. Ejecuciones presupuestales de los municipios del AOA en 2013.

\begin{tabular}{|c|c|c|c|c|c|c|c|c|c|}
\hline $\begin{array}{l}\text { Agregados } \\
\text { financieros }\end{array}$ & $\begin{array}{l}\frac{}{8} \\
\frac{0}{0} \\
\frac{0}{x}\end{array}$ & 을 & 竞 & త్ర & 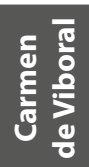 & 恶 & 苫 & 总 & כ. \\
\hline $\begin{array}{l}\text { 1. Gastos de } \\
\text { funcionamiento }{ }^{2}\end{array}$ & 17.00 & 25.54 & 26.26 & 41.83 & 48.78 & 53.62 & 50.62 & 30.25 & 55.83 \\
\hline $\begin{array}{l}\text { 2. Respaldo del } \\
\text { servicio deuda }\end{array}$ & 2.69 & 8.11 & 6.73 & 7.79 & 4.17 & 11.44 & 5.61 & 6.52 & 8.62 \\
\hline $\begin{array}{l}\text { 3. Dependencia de } \\
\text { las transferencias }{ }^{4}\end{array}$ & 21.77 & 25.02 & 42.49 & 39.24 & 41.36 & 45.47 & 60.55 & 63.10 & 72.21 \\
\hline $\begin{array}{l}\text { 4. Generación de } \\
\text { recursos propios }\end{array}$ & 92.97 & 85.15 & 84.85 & 80.98 & 72.54 & 75.00 & 72.18 & 31.66 & 63.61 \\
\hline $\begin{array}{l}\text { 5. Magnitud de la } \\
\text { inversión }^{6}\end{array}$ & 90.84 & 82.15 & 83.91 & 82.84 & 81.16 & 77.22 & 85.59 & 87.17 & 82.28 \\
\hline $\begin{array}{l}\text { 6. Capacidad de } \\
\text { ahorro }^{7}\end{array}$ & 83.03 & 71.68 & 71.22 & 61.67 & 52.58 & 50.16 & 51.92 & 67.50 & 47.03 \\
\hline $\begin{array}{l}\text { Índice de } \\
\text { desempeño fiscal }^{8}\end{array}$ & 90.73 & 84.72 & 82.39 & 80.40 & 77.65 & 75.03 & 74.93 & 70.42 & 69.82 \\
\hline
\end{tabular}

Fuente: elaboración propia a partir de datos del DNP, datos 2013 reportados en 2014

2 Autofinanciación de los gastos de funcionamiento = Gasto funcionamiento/ ICLD* $100 \%$.

3 Respaldo del servicio de la deuda $=$ Servicio de la deuda /ingreso disponible $* 100 \%$. 4 Dependencia de las transferencias de la Nación y las Regalías = Transferencias + Regalías/ingresos totales * $100 \%$.

5 Generación de recursos propios = Ingresos tributarios /ingresos corrientes * $100 \%$. 6 Magnitud de la inversión = Inversión / gasto total $* 100 \%$.

7 Capacidad de ahorro = Ahorro corriente / ingresos corrientes * $100 \%$.

8 Indicador de desempeño Fiscal: Variable que resume los 6 indicadores anteriores en una sola medida, con escala de 0 a 100. 
La tabla 2 muestra la composición de las seis variables por cada uno de los municipios, las cuales determinan el indicador de desempeño fiscal. De ellas se presentan los siguientes resultados:

- Gasto de funcionamiento: se observa cómo, de los nueve municipios, La Unión es el que presenta la variable peor calificada, con unos gastos de funcionamiento de 55.83, seguido de Marinilla y El Santuario con variables valoradas en 53.62 y 50.62 , respectivamente. No muy alejado de los anteriores reportes, El Carmen de Viboral es el que obtiene el cuarto lugar entre los municipios mal valorados en cuanto a su gasto de funcionamiento, con un indicador de 48.78, seguido de los municipios de La Ceja y San Vicente, con variables de 41.83 y 30.25 , respectivamente. Por su parte, el municipio de Rionegro es el que mejor variable reporta, con un indicador de 17.00, seguido de El Retiro, que posee un indicador de 25.54, y Guarne, con 26.26.

- Respaldo del servicio de la deuda: este componente fiscal refleja que los municipios que reportan los mejores resultados en cuanto a la magnitud de la deuda son Rionegro, El Carmen deViboral y EI Santuario, con unas variables, según la información reportada, valoradas en 2.69, 4.17 y 5.61, respectivamente. Para los demás municipios, el indicador no es tan bueno; sin embargo, se puede ver que todos tienen niveles de endeudamiento bajos, ya que EI Santuario, San Vicente, Guarne y La Ceja presentan valores para este componente de 6.52, 6.73 y 7.79, respectivamente. Y en el caso de los municipios de El Retiro, La Unión y Marinilla, se presentan índices más altos valorados en $8.11,8.62$ y 11.44; esto implica que sus ingresos son proporcionalmente menores que los ingresos de los otros municipios.

- Dependencia de las transferencias: en esta variable se observa que las finanzas de los municipios de La Unión, San Vicente y El Santuario podrían verse comprometidas por poseer las peores calificaciones: $72.21,63.10$ y 60.55 , respectivamente. Les siguen los municipios de Marinilla, Guarne, El Carmen de Viboral y La Ceja, los cuales poseen unos resultados intermedios considerados en unos índices que corresponden a 45.47, 42.49, 41.36 y 39.24, respectivamente. Por otra parte, para los municipios de Rionegro y El Retiro se vislumbra su capacidad de gestionar recursos propios, en los cuales las transferencias pesan en baja proporción; así lo indican los resultados estimados en 21.77 y 25.02 , que se encuentran, en cierta magnitud, holgados en comparación con los índices de sus vecinos regionales.

Según los resultados que arroja este análisis, se puede inferir que estos índices son acordes con lo que establece la ley en relación con la 
destinación de dineros de diferentes niveles del Gobierno a municipios de cuarta, quinta y sexta categoría. Como se muestra en la tabla 1, es de notar que todos los municipios de esta región se clasifican en las categorías $4^{a}$, $5^{\mathrm{a}}$ y $6^{\mathrm{a}}$, a excepción de Rionegro, municipio de $2^{\mathrm{a}}$ categoría, lo cual que coincide con los buenos resultados en sus diferentes variables analizadas.

Por lo anterior es que los municipios especialmente clasificados en $6^{a}$ categoría, como La Unión, San Vicente y El Santuario, poseen una alta captación de dinero proveniente de los diferentes niveles de gobierno; ello indica un exceso de dependencia y una financiación de la administración principalmente con dichas transferencias. Según esto, se hace pertinente citar a José Lenin Galindo Urquijo (comunicación personal, 25 de junio de 2014), quien menciona que el modelo de descentralización aplicado en Colombia es en algunos casos perverso, ya que pareciera premiar a los municipios que son pobres y tienen mucha población; es decir que, dentro de la distribución del SGP, en la medida en que se posea una mayor población y se tengan mayores Necesidades Básicas Insatisfechas (NBI), las entidades subnacionales giran más dinero, lo cual genera pereza fiscal y desincentivo para el recaudo de sus recursos propios.

- Generación de recursos propios: los indicadores evidencian rezagos en la gestión tributaria de ciertos municipios; es el caso del municipio de San Vicente, con una variable de 31.66. Por su parte, los municipios de La Unión, El Santuario, El Carmen de Viboral y Marinilla representan un nivel más alto en consideración al recaudo tributario que corresponde a $63.61,72.18,72.54$ y 75.00 , respectivamente. En ese sentido, se destaca la gestión de los municipios de La Ceja, Guarne, El Retiro y Rionegro, que poseen las mejores calificaciones en esta variable, de acuerdo con su ejecución $(80.98,84.85,85.15$ y 92.97 , respectivamente). Es así como en este indicador recae el mayor análisis en cuanto al índice agregado del desempeño fiscal, pues de este balance parte la destinación para los diferentes agregados financieros; traducido esto, se dice que se ejecuta en gasto de funcionamiento, respalda las acreencias financieras, se genera inversión y finalmente, después de cubrir estos compromisos, se espera un excedente en función de reserva para cubrir situaciones de crisis o inviabilidad financiera.

- Magnitud de la inversión: los gastos de inversión -que permiten incrementar la capacidad de producción y la productividad en el campo de la infraestructura física, económica y social- se evidencian en los municipios de Rionegro, San Vicente y El Santuario, debido a que conforman el mayor volumen de este aspecto en la región, con unos índices valorados en $90.84,87.17$ y 85.59 , respectivamente. 
Como se observa en la tabla 2 y se expresa en el párrafo anterior, en las estadísticas aparece San Vicente, municipio que en determinadas variables no cumple satisfactoriamente con lo esperado (recursos propios) para obtener una posición dentro de los mejores rankings; pero se resalta el índice de destinación de recursos para inversión, representado por un valor de \$10.118.000.000 (diez mil ciento dieciocho millones de COP); es decir, el municipio no deja de hacer esfuerzos en inversión que se traduzcan en el bienestar de sus habitantes.

Por otra parte, en menor medida se encuentran los municipios de Guarne, La Ceja y La Unión, con unos valores de 83.91, 82.84 y 82.28 correspondientes a cada municipio. Para efectos del análisis, se deduce que, en términos inversionistas, la ejecución de inversión en el bienestar de la comunidad se encuentra en excelentes condiciones. En cuanto a los demás municipios, los cuales no están muy alejados de los anteriores reportes, se determinan unos índices considerables en materia financiera para el caso del municipio de El Retiro, el cual reporta el séptimo lugar entre estos, con un indicador de 82.15, seguido de los municipios de El Carmen de Viboral y Marinilla, con variables de 81.16 y 77.22, respectivamente.

- Capacidad de ahorro: al observar el comportamiento de los índices, los municipios que poseen la peor calificación en la variable capacidad de ahorro son La Unión, Marinilla y El Santuario con unos indicadores del 47.03, 50.16 y 51.92. Cabe resaltar que esto no indica que las entidades territoriales se encuentren en estado de desahorro, salvo el municipio de La Unión pues se encuentra con una magnitud por debajo de lo esperado. En lo que respecta a los municipios de El Carmen de Viboral, La Ceja y San Vicente, estos presentan unos valores de $52.58,61.67$ y 67.50 , respectivamente. Por su parte, el municipio de Guarne reporta un valor, en secuencia a los anteriores, de 71.22, seguido de El Retiro, que posee un indicador de 71.68 y Rionegro con 83.03 .

Teuken Bidikay felicita al Colegio de Contadores, Economistas y Administradores del Uruguay en sus 125 años de trabajo por la integración profesional en beneficio de la sociedad uruguaya.

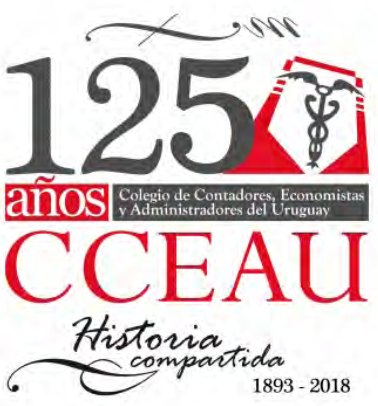


En el siguiente gráfico, se muestran los anteriores resultados:

Gráfico 1: Variables del Indicador de Desempeño Fiscal de los municipios del AOA en 2013 Variables del Indicador de Desempeño Fiscal de los municipios del
AOA en 2013

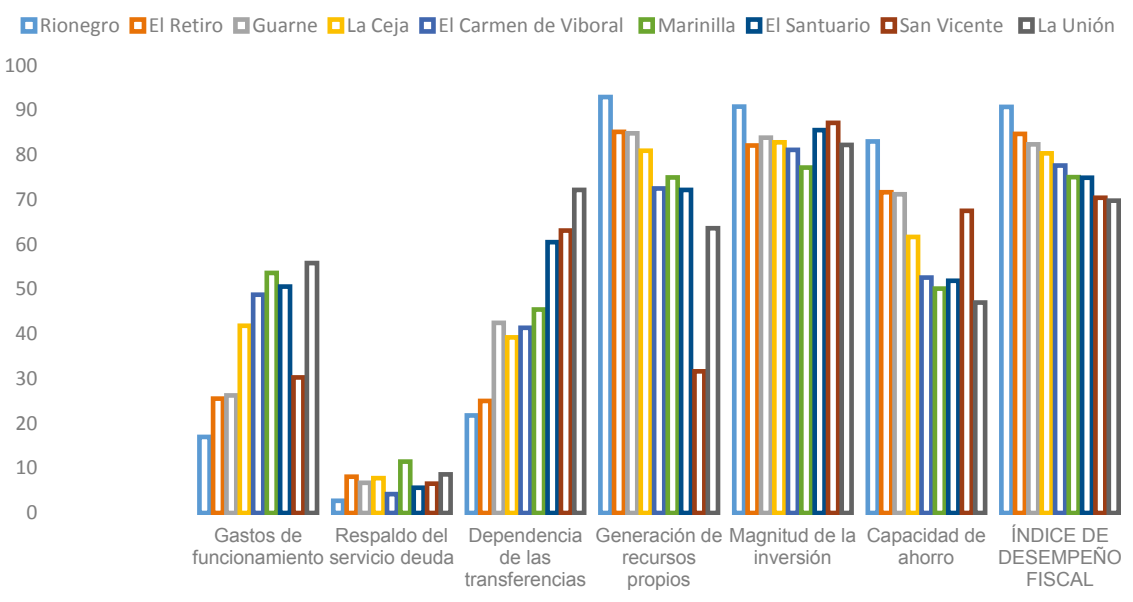

Fuente: Elaboración propia a partir de los datos de las ejecuciones presupuestales para los municipios del AOA en 2013

Los anteriores resultados permiten establecer algunas conclusiones y recomendaciones que aparecen a continuación. Con ellas se espera que las entidades territoriales implementen algunas acciones no solo para mejorar su posicionamiento en el ranking de desempeño fiscal, sino también para mejorar su tipología de viabilidad financiera; esto se traduciría en un mayor progreso y sostenibilidad de la calidad de vida de sus habitantes.

\section{Conclusiones y recomendaciones}

Las conclusiones y recomendaciones que a continuación se presentan propenden por el mejor desempeño de las seis variables contenidas en el indicador sintético de desempeño fiscal, con miras al mejoramiento de las finanzas públicas locales y la viabilidad financiera de las entidades territoriales. No obstante, es preciso anotar que los siguientes son consejos referidos a buenas prácticas fiscales a nivel general, ya que para especificar recomendaciones a cada uno de los municipios se haría necesario un estudio más profundo de la realidad productiva, cultural, sociodemográfica y económica particular de cada uno de ellos. 


\section{En relación con los gastos de funcionamiento}

Las entidades territoriales deben buscar el logro de bajos niveles en materia de gastos de funcionamiento. Para lo anterior deben estructurar sus procesos y procedimientos administrativos por medio de la implementación de manuales de funciones, disminución de trámites y eficiencia en las operaciones de los funcionarios públicos. Adicionalmente, los municipios deben considerar el hecho de que los gastos de funcionamiento solo hacen alusión a aquellos de orden administrativo, ya que las labores propias de los procesos misionales podrían entenderse como gasto de capital o inversión y, por tanto, es en estos proyectos donde los municipios deben contratar a los funcionarios para no reflejar esto dentro de los gastos de funcionamiento.

En este sentido, el municipio de Rionegro es un referente a nivel nacional en el ranking de desempeño fiscal, porque para el año 2000 poseía un total de 517 funcionarios públicos, mientras que para el año 2014 ya poseía únicamente 232 (García, comunicación personal, 19 de diciembre de 2014), demostrando esto eficiencia en la contratación, mejoramiento en los procedimientos y procesos y disminución en el gasto de funcionamiento, aun siendo un municipio certificado en salud, educación, saneamiento básico y agua potable.

Continuando con el análisis de esta variable y haciendo énfasis en que una de las claves de éxito para cualquier municipio es que mantenga controlados los gastos de funcionamiento, es necesario que las entidades territoriales dispongan de procedimientos claros y busquen que los procesos sean certificados con el fin de tener más eficiencia, eficacia y calidad en la prestación del servicio. En caso de que el número de empleados sea muy alto, la prestación de servicios puede ser anacrónica, pero si se tienen pocos se tiene deficiencia y debilidad en esta materia. En este sentido, los municipios deben profesionalizar las plantas de cargos y buscar que los funcionarios dispongan de competencias apropiadas; esto permite que el conocimiento que adquieran los funcionarios sea aplicado en los puestos de trabajo. El cambio permanente de los funcionarios hace un daño grande a la gestión pública, ya que las personas que recién entran no se han apropiado de los procesos, por lo que es recomendable propender por una mayor permanencia de los funcionarios que han tenido un desempeño óptimo en sus puestos de trabajo.

Es importante, además, tener en cuenta que cuando los municipios mejoran su categoría, según los criterios establecidos en la Ley 617 de 2000 , es posible que tengan nuevas responsabilidades y nuevas funciones -como, por ejemplo en materia de salud y de educación-. Las 
entidades territoriales deben, por tanto, propender por tener estructuras más fuertes y sólidas para responder a las nuevas funciones y no asumir nuevas competencias para las que la estructura administrativa no está preparada.

\section{En relación con la deuda pública}

Uno de los factores fundamentales a tener en cuenta es la calificación de riesgo, que es otorgada tanto a las entidades del orden central como a las descentralizadas, porque es esencial para la determinación del nivel de confianza tanto de consumidores como de inversionistas, quienes jalonan el nivel de desarrollo local y nacional. Adicionalmente, los municipios deben tratar que la deuda que posean sea en pesos; de lo contrario tendrían que buscar mecanismos de coberturas de tasa de cambio para evitar estar expuestos a la fluctuación y a la coyuntura internacional.

Por su parte, los municipios deben buscar que sus niveles de deuda sean sostenibles y que verdaderamente se soporte en los recursos que se pueden pignorar, mas no en ingresos inflados que apalanquen una mayor deuda y que, posteriormente, generen niveles de sobrendeudamiento. Otro aspecto que perjudica actualmente a las entidades territoriales y que termina configurándose como deuda pública son los pasivos pensionales, ya que el artículo 72 de la Ley 617 de 2000 los constituye así, una vez cumplidos los requisitos para hacerse exigibles.

Otra de las recomendaciones es que las entidades territoriales realicen una evaluación periódica de sus niveles de deuda, además de velar porque los Consejos de Política Fiscal (CONFIS) cumplan la función para la que están creados, que no es más que hacer una evaluación del estado económico de cada una de las entidades descentralizadas y que los indicadores sean apropiados y aplicados.

Por último, es importante señalar que muchas veces los municipios se endeudan por falta de gestión administrativa, porque no presentan proyectos, por ejemplo, ante el ministerio respectivo; mediante esta estrategia podrían obtener recursos del $30 \%, 50 \%$ e incluso del $100 \%$ del valor del proyecto. De esta manera el municipio podría recurrir a deuda, pero en una menor cuantía, o salir a endeudarse sin cerrar la brecha de la evasión de los impuestos, lo que se traduce en baja o falta de gestión, que permite que los ciudadanos se queden con los recursos que tendrían que pagar por concepto de impuestos y aparte se generan intereses a la entidad territorial. Bajo estos casos, estar endeudado no es bueno; por tanto, lo que se recomienda es ejecutar más proyectos con recursos de cofinanciación o cooperación y, además, aplicar mayor control sobre la evasión de impuestos. 


\section{En relación con la dependencia de las transferencias de la Nación}

Los municipios deben tratar de tener el menor grado de dependencia de las transferencias de la Nación por varias razones, a saber: aunque los recursos sean de orden constitucional para fines específicos, para sectores estratégicos que contribuyen al desarrollo del país -como la salud, la educación, el saneamiento básico, el agua potable-, también hay otras necesidades territoriales que no se cubren con estos recursos y que, por tanto, sí se propende por una mejor calidad de vida local, requerirían el mayor recaudo de recursos propios.

En este sentido, la clave es fortalecer los ingresos propios. Para esto los municipios deben desarrollar políticas que permitan, en primer lugar, reconocer cuáles son los factores claves de éxito para el desarrollo industrial, comercial y de servicios; en segundo lugar, desarrollar encadenamientos productivos para tener empresas perdurables en el tiempo que generen tributación y que además generen empleo para los habitantes del territorio.

\section{En relación con la generación de recursos propios}

La Ley 14 de 1983 (Congreso de la República, 1983) ordena actualizaciones catastrales cada cinco años para tener actualizada la base gravable de la principal fuente de recursos propios para muchos de los municipios colombianos, que es el impuesto predial. No obstante, algunas entidades territoriales no las realizan exactamente con esta periodicidad, lo cual genera pérdida de eficiencia en este recurso debido a que sus predios podrían estar subvalorados y de esta manera estar privando al municipio de cifras representativas en sus ingresos tributarios.

Muchos de los municipios no cuentan con la estructura adecuada para el desarrollo de procesos de fiscalización tributaria, lo que hace que en muchas ocasiones dejen de percibir recursos debido a la evasión, elusión o mora en el pago de los tributos. Asimismo, se percibe que muchos de los registros mercantiles de las diferentes entidades territoriales no están en línea y los estatutos de renta no siempre están actualizados. Todos estos factores han generado pérdidas de recursos propios para las entidades territoriales, que sería un factor fácil de resolver toda vez que se debe a un esfuerzo de implementación y eficiencia de estructuras de control y fiscalización de rentas.

Adicionalmente, según Juan Alberto García García (comunicación personal, 19 de diciembre de 2014), parte de la ineficiencia fiscal se debe a municipios altamente burocratizados, donde se cree que generar empleo es función del Gobierno, cuando el deber del Estado es ser promotor de 
empleo y pasar del clientelismo al empresarismo para generar los factores que realmente generen empleo en los municipios.

Por su parte, las entidades territoriales deben enfocar el presupuesto hacia la realización de obras y acciones que generen desarrollo; es decir, deben estar enfocadas en cumplir principalmente dos funciones: disminuir las Necesidades Básicas Insatisfechas (NBI) y generar crecimiento económico. En este sentido, si un gobierno no es capaz de generar ingresos en montos superiores a los que registran el crecimiento de la inflación y del salario y al crecimiento de su curva demográfica, lo que está generando es más pobreza para sus ciudadanos. En este aspecto es donde se refleja la verdadera gestión del gobernante.

\section{En relación con otras variables en general}

Ya se enunciaba que la principal clave para fortalecer las finanzas públicas es lograr identificar el sector productivo de éxito y estratégico para el desarrollo de un municipio. De allí deben partir los esfuerzos que se dirigen al mejoramiento de la viabilidad financiera de las entidades territoriales, y para esto podrían desarrollarse, con visión emprendedora, procesos de formación alrededor del sistema productivo.

De otra parte, es preciso propender por que el presupuesto territorial cumpla con las funciones ya enunciadas de disminución de las NBI y que en efecto se contribuya al desarrollo local, porque el éxito de la ejecución presupuestal no se mide por los recursos gastados o recaudados según lo planeado, sino por el impacto que estos recursos generen en la comunidad. En términos generales, los municipios no solo del AOA, sino de todo el país, deberán considerar como prioridad acciones tales como la aplicación de las actualizaciones catastrales en los periodos establecidos por la ley, cerrar la brecha de la evasión de impuestos, racionalizar el gasto y orientar la inversión a sectores clave del desarrollo municipal.

\section{Posta del Chuy}




\section{Referencias bibliográficas}

- COLOMBIA. Congreso de la República. (1983). Ley 14 de 1983. Por la cual se fortalecen los fiscos de las entidades territoriales y se dictan otras disposiciones. Bogotá D.C.: Diario Oficial. 36.288.

- COLOMBIA. Congreso de la República. (1997). Ley 358 de 1997. Por la cual se reglamenta el artículo 364 de la Constitución y se dictan otras disposiciones en materia de endeudamiento. Bogotá D.C.: Diario Oficial. 42.973.

- COLOMBIA. Congreso de la República. (2003). Ley 819 de 2003. Por la cual se dictan normas orgánicas en materia de presupuesto, responsabilidad y transparencia fiscal y se dictan otras disposiciones. Bogotá D.C.: Diario Oficial. 45.243.

- COLOMBIA. Ministerio de Hacienda y Crédito Público. (2000). Ley 617 de 2000. Por la cual se reforma parcialmente la ley 136 de 1994, el decreto extraordinario 1222 de 1986, se adiciona la ley orgánica de presupuesto, el decreto 1421 de 1993, se dictan otras normas tendientes a fortalecer la descentralización y se dictan normas para la racionalización del gasto público nacional. Disponible en internet: http://www.minhacienda.gov.co/portal/page/portal/HomeMinhacienda/ asistenciaentidadesterritoriales/Publicaciones/Libros/Ley\%20617\%20de\%20 200\%20version\%202008.pdf Consultado 12.08.2014.

- COLOMBIA. Departamento Nacional de Planeación (2010). Informe de desempeño fiscal de los departamentos y municipios 2010. Bogotá: DNP. Disponible en internet: https://colaboracion.dnp.gov.co/CDT/Desarrollo\%20 Territorial/Desempe\%C3\%B10\%20Fiscal\%202010.pdf. Consultado 21.09.2014

- COLOMBIA. Departamento Nacional de Planeación (2011). Desempeño fiscal de los departamentos y municipios 2011. Bogotá: DNP. Disponible en internet: https://colaboracion.dnp.gov.co/CDT/Desarrollo\%20Territorial/ Desempe\%C3\%B1oFiscaL_2011_CEVC2_WEB.pdf Consultado 10.10.2014.

- COLOMBIA. Departamento Nacional de Planeación (2012). Desempeño fiscal de los departamentos y municipios 2012. Bogotá: DNP. Disponible en internet: https://colaboracion.dnp.gov.co/CDT/Desarrollo\%20Territorial/Informe\%20 Desempe\%C3\%B10\%20Fiscal\%202012.pdf Consultado 06.08.2016

- COLOMBIA. Departamento Nacional de Planeación (2013). Desempeño fiscal de los departamentos y municipios 2013. Bogotá: DNP. Disponible en internet: https://colaboracion.dnp.gov.co/CDT/Desarrollo\%20Territorial/Documento\%20 Desempe\%C3\%B10\%20Fiscal\%202013.pdf Consultado 09.08.2016

- GALINDO, José Lenin. (2014). Entrevista asesor de la Dirección de Desarrollo Territorial del DNP. 25 de junio de 2014. Rincón, C. \& Restrepo, A. Entrevistadoras.

- GARCÍA, Juan Alberto. (2014). Entrevista secretario de Hacienda del municipio de Rionegro, 19 de diciembre de 2014. Rincón, C. \& Restrepo, A. Entrevistadoras.

\begin{tabular}{|c|l|}
\hline $\begin{array}{c}\text { Para citar } \\
\text { este artículo: }\end{array}$ & $\begin{array}{l}\text { Rincón, C. \& Restrepo, A.L. (2017). Indicador de } \\
\text { desempeño fiscal: análisis de los resultados de su } \\
\text { aplicación en los municipios del altiplano del oriente } \\
\text { antioqueño. Teuken Bidikay Vol. } 8 \text { N¹1. Pp. 61-79 }\end{array}$ \\
\hline
\end{tabular}


Ubicadoen Lavalleja, a $8 \mathrm{~km}$. de Minas, la ciudad capital departamental, el Valle del Hilo de la Vida debe su nombre al recorrido que realiza el agua por el valle, en forma de cascada, desde una fuente de agua que surge del subsuelo. El sitio arqueológico, de más de 1200 metros de longitud, presenta cerca de 90 montículos de piedras, que van desde un metro hasta los tres metros y medio de altura, todos orientados hacia el poniente y ubicados en el lado cóncavo del valle. Formados por lajas de piedra chatas, pequeñas, y sin ningún material que las una, hacen parte de una estructura que se ha mantenido en pie por más de mil años y se especula que fueron construidos por Charrúas, Guenoas o Minuanos, tribus aborígenes que poblaban esta región. Los historiadores coinciden en que estas enigmáticas construcciones fueron tratadas como vórtices de energía y usadas en ceremonias religiosas por los indígenas. Seguir el Hilo de la Vida invita a experimentar sensaciones que combinan naturaleza, misterio, historia y cultura, en un paraje donde la voz del pasado se escucha en una perfecta caja de resonancia.

Eduardo Valera.

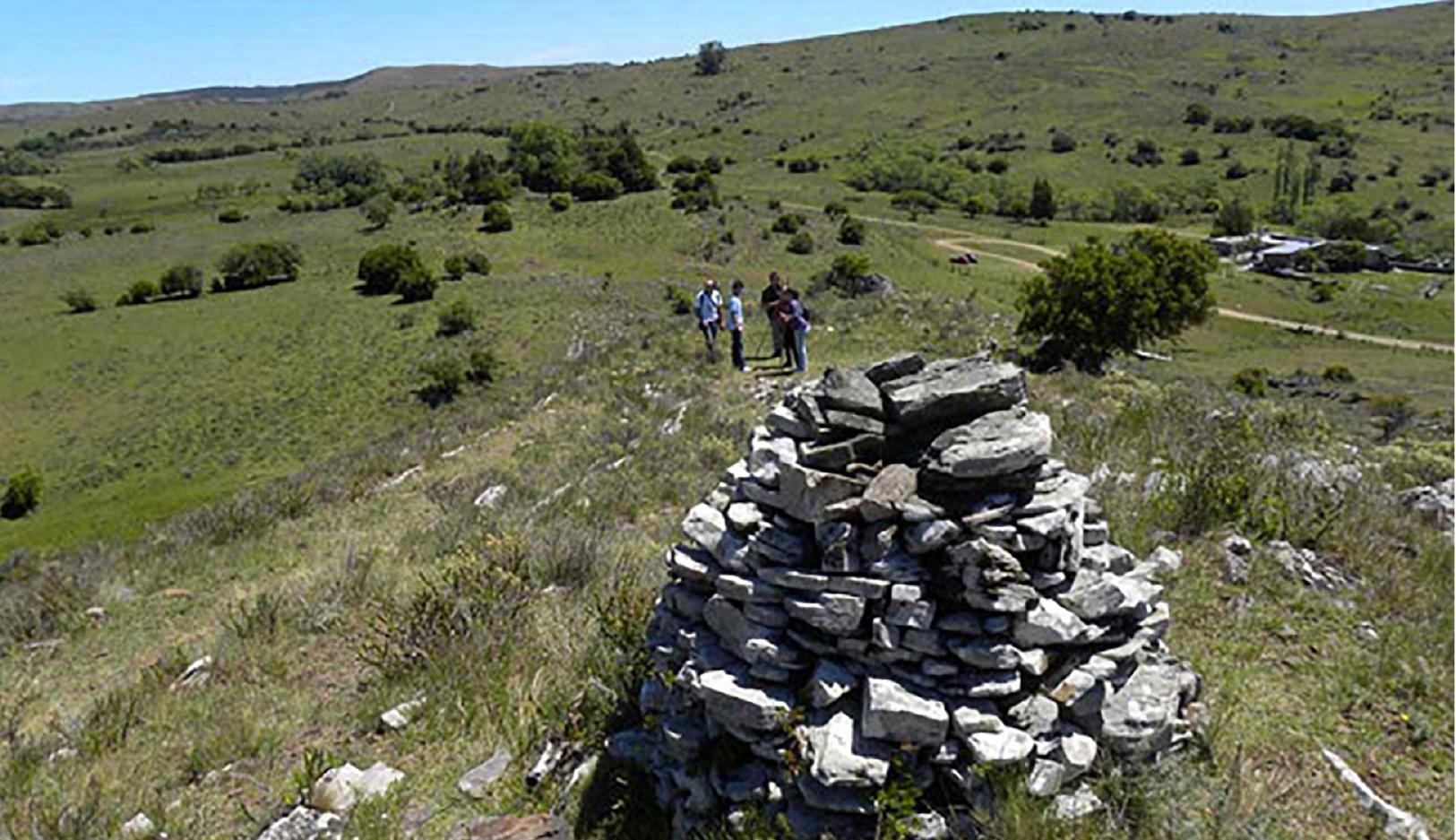

